\title{
Brain-Controlled for Changing Modular Robot Configuration by Employing Neurosky's Headset
}

\author{
Muhammad Haziq Hasbulah ${ }^{1}$, Fairul Azni Jafar ${ }^{2}$, Mohd. Hisham Nordin ${ }^{3}$, Kazutaka Yokota ${ }^{4}$ \\ Faculty of Manufacturing Engineering, University Teknikal, Hang Tuah Jaya, 76100, Durian Tunggal, Melaka, Malaysia ${ }^{1,2,3}$ \\ Research Div. of Design and Eng. for Sustainability, Graduate School of Engineering, Utsunomiya University ${ }^{4}$ \\ 7-1-2 Yoto, Utsunomiya-shi, 321-8585, Japan
}

\begin{abstract}
Currently, the Brain Computer Interfaces (BCI) system was designed mostly to be implemented for control purpose or navigation which are mostly being employed for mobile robot, manipulator robot and humanoid robot by using Motor Imagery. This study presents an implementation of BCI system to Modular Self-Reconfigurable (MSR) Dtto robot so the robot able to propagate multiple configurations based on EEGbased brain signals. In this paper, a Neurosky's Mindwave Mobile EEG headset is being used and a framework of controlling the Dtto robot by EEG signals, processed by OpenViBE software are built. The connection being established between Neurosky's headsets to the OpenViBE, where a Motor Imagery BCI is created to receive and process the EEG data in real time. The main idea for system developed is to associate a direction (Left, Right, Up and Down) based on Hand and Feet Motor Imagery as a command for Dtto robot control. The Direction from OpenViBE were sent via Lab Streaming Layer (LSL) and transmitted via Python software to Arduino controller in the robots. To test the system performance, this study was conducted in Real time experiments. The results are being discussed in this paper.
\end{abstract}

Keywords-Dtto robot; motor imagery; OpenVibe; modular robot; configuration; communication

\section{INTRODUCTION}

In 1970s, a system for communication between human brain and external application being established by Jacques where the raw data obtained based on human brain signals being manipulated into a data vector [1]. The system basically revolves around an electroencephalogram (EEG) which is the brain electrical field measurement where it is being acquired by electrode (head placement) during tasks performing and widely used for diagnosis of mental disease and research in bioengineering [2].

One of the main motivations of BCI research is to help the disabled or elderly. According to the World Health Organization (WHO), 15 million people suffer stroke every year, with one third of them left permanently disabled [3]. Anyhow, with the advancement of technologies nowadays, the BCI implementation has been comprehend for individuals who non-disabled or at normal conditions. The extended BCI implementation is in 3D virtual environment, gaming, research, entertainment and in robotic study. Basically, the robots normally being controlled by using a typical input device for robot control which could be a mouse, a keyboard or a joystick by a normal healthy person. Same goes to Modular Self- reconfigurable (MSR) robot which being controlled by conventional input interfaces. Unfortunately, that kind of input interfaces is inconvenient for people who have physical disabilities where most of the cases the person is unable to use their arm, unable to walk or even speak. Hence, the person who in that condition unable to effectively use the robot by the conventional input interfaces. This is the cases where the braincontrolled robot is being very useful. The development of brain-controlled robots would be very useful in such cases and this implementation has a potential to be employed to MSR robot. As a robot could be sent to places that inaccessible to humans, MSR robot is one of the robot which can used and it is possible to be controlled by brain.

Now-a-days, BCI system design is based on the exploitation of three categories of brain signals, which include event related desynchronization/event related synchronization (ERD/ERS), steady state visually evoked potential (SSVEP), and P300. There are certain disadvantages and advantages of the brain signals mentioned. Basically, the signals acquired based on the external stimulations of the user and the electrode positions on the head scalp.

In robotic field, BCI system that exploits the ERD/ERS brain signals basically being developed in order for robot control with EEG signals recorded during mental task performance, such as Motor Imagery (MI) and Motor Execution (ME) [4]. ERD/ERS control signals does not require any external stimulus. Hence, users or subject only need to focus their whole attention on controlling the MSR robot.

Even though, the other brain signal type able to be manipulated without substantial training, and it has capabilities to provide a several number control command possibilities, according to Al-Neheimish [5] cited by Malki et al. [6], Motor Imagery provides results with faster feedback. It is due to the asynchronous type of BCI system (ERD/ERS) which independent to the cue-based manner unlike synchronous BCI system (P300 and SSVEP). It is because this system manner make it possible for the EEG features to be process without waiting for the instruction to be completed as it can be process one after another [7][8][9][10]. However, the ERD/ERS BCI system also can be operated based on Cue-based system. Besides that, cue based Motor Imagery (MI) BCI is suitable for our research because it can be used without muscle involvement which is the purpose of BCI system and it gives a certain period for execution of the MSR robot to change configuration. 
MSR robot capability for having multiple configurations is being tested by MI BCI system in this study. It acquires EEG signals from human brain and classifies them into two user mental states, left-hand and right-hand motor imagery. To ensure MSR robot is able to propagate multiple configurations based on MI BCI system, another two mental states for the user will be classified so the system will have 4 control input for the robot to change its configuration into 4 types of configurations. Besides that, the Neurosky Mindwave Mobile EEG headset is being used to obtain the EEG signals from the user. It is only have 1 electrode and a non-invasive EEG acquisition device. An expensive EEG headset device was mostly being considered in order to exploiting ERD/ERS brain signals because of the number of electrodes which is more and based on 10-20 electrode positions.

As a preliminary framework that this paper describes, the system of MI BCI system is capable of operating independently and being incorporated into larger platform which is controlling MSR robot. The capabilities of Neurosky for incorporated with MI BCI is expected to produce a poor result as Neurosky only have 1 electrode, meanwhile for MI system, the EEG data has to be acquired by multiple electrodes, positioned based on 10-20 electrode placement system. However, this study is being to show and prove that MSR robot can be controlled and changed for multiple configurations through MI BCI system by communication establishment from OpenViBE to Python for robot control. Hence, since the efficiency for the MSR robot to change its configuration based on the EEG signal is low, this study will not being concluded based on the signal accuracy only. This study will be concluded based on the results of communication establishment based on MI BCI system. Therefore, this study will provide a new perspective for the types of robot to be controlled by EEG.

In the next sections onwards, this paper discussed about the background for each one of the necessary element implemented to achieve this study objectives. Then, there is Section III where this experimental methodology for this study is being discussed. The system performance result is presented and discussed in Section IV and Section V is the conclusion drawn from the result of this study. Discussion on future work is being discussed in Section VI.

\section{BACKGROUND}

\section{A. Electroencephalogram (EEG)}

Billions number of neurons made up the brains where each of them is connected to thousands of nerves by axons. As an action potential merged in the dendrites, a stimulus is conveyed through axons in order to communicate. Hence, an electrical field will be generated based on synaptic current produced. According to Ala et al. [11], EEG is a technique for neural sources being localized with a high time resolution as electric scalar measurements is being exploited. Besides that, as researcher involved in EEG study, The International 10-20 standard is need to be known as it is used for positioning electrode on the user head scalp and multiple channels are used because the EEG signals are difference both in term of temporally and spatially.
The electrical signal generated by a single neuron in the human nervous system is too small to be assessed by an electrode on the head scalp [12]. Hence, the measurement of electrical activity is able to be recorded as it comes from the summation of the activity of hundreds of neurons with the electrode based on the closeness of electrode position to the head scalp. Neurons in a group electrify more by collectively and reflecting immediately as a signal being measured by becoming stronger and more collectively to the electrify frequency [12]. The value of the electrical field measured which produced by a group of neuron as overall is known as EEG signal [13].

\section{B. Neurosky's Mindwave Mobile}

Neurosky's [14] device is one of the commercialized EEG devices which being developed to have the acquisition of the EEG based on one-channel of electrode. The electrode position for this device is designed to be at user forehead for frontal recording. Besides that, the type of electrode being used for this device is dry electrode. Reference point of Neurosky is being placed at the earclip. The EEG data from Neurosky transmitted wirelessly using Bluetooth. This device targeting low-end consumer market as it is low cost. Neurosky's headset is used to acquire the EEG data from user head scalp in this study. In Fig. 1, Neurosky's headset and the position of Neurosky's electrode based on 10-20 system are shown.
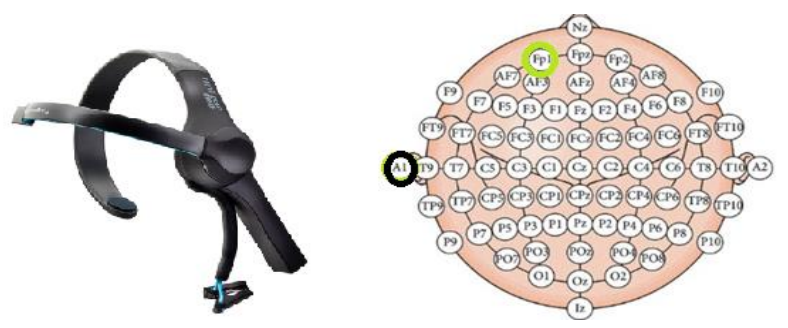

Fig. 1. Neurosky Mindwave Mobile (Left) [14] and the 10-20 System with the Neurosky Electrode Placement in Green-Colored Circle (Right).

\section{OpenViBE}

An EEG processing software known as Open ViBE is a free to be downloaded software as it is an open source platform. This software is being developed so that it can be used for designing and testing of the BCI system. Besides that, it is a software where the developed BCI system can be used in real-time event. This software can be used for processing the EEG brain signals from signal acquisition, feature extraction, classification and this software also capable to visualize the data in real time [15]. This software provides a virtual display for convenient user interaction and can be connected with various EEG headsets for data acquisition. In addition, this software also provided with Classifier Trainer box which will be used for training models to classify input data. The available classifiers in OpenVibe processing tools are LDA, SVM and MLP [16].

\section{Dtto-Modular Self-Reconfigurable Robot}

Hybrid-type of MSR robot known as M-TRAN is being a concept example for the development of Dtto MSR robot. Dtto robot is being designed in order to provide free space in half of the robot as much as possible. It is because so that it can be used by users to set up their preferred sensor such as Infrared 
(IR) sensor or install more actuators. It is $3 \mathrm{D}$ printable and at low cost. Dtto is a robot module being designed which consists of two boxes, rounded on one side, connected by a bar. Multiple Dtto robot can join with each other by magnet attraction and able to propagate multiple configuration. It can be fully printed with $3 \mathrm{D}$ printer and has been designed using FreeCad software. The robot communicate to each other by Bluetooth and radio communication. Dtto Modular robot built with the idea of male and female part [17].

\section{E. Event-Related Desynchronization/Event-Related Synchronization (ERD/ERS)}

ERD/ERS is one of the brain signal which commonly being implemented for the BCI system. It is generated when mental tasks are being performed such as by Mental Arithmetic or Motor Imagery (MI) [18]. It is highly frequency band specific where ERD/ERS is able to be displayed at different locations on the head scalp [19]. Actually, there is a slightly differences between ERD and ERS. ERD happened to exhibit power of frequency band in a decreasing manner and meanwhile, ERS happened to exhibit power of frequency band in an increasing manner. The differences for the power spectrum of frequency bands generated show that there are changes occur for a group of neurons in firing patterns which happened during, before or after a motor task event. According to Manca [20], ERD/ERS generated as there are specific frequency changes where the amplitude of the brain signals is based directly on the number of synchronously active neurons. Hence, the increase and decrease for the power spectrum of frequency band can be considered as synchronization and desynchronization. The correlations of synchronization and desynchronization of cortical rhythms indicated to be as ERD/ERS [21]. Analysis of ERD/ERS brain signals recorded has to be done in frequency domain, where the frequency band responds prior to movement. According to Durka [22], the classical evaluation of ERD/ERS have categorized the brain signal into three frequency bands which are mu-band (10-12 Hz), beta-band (14-30 Hz) and gamma-band (30-40 Hz). Decreasing and increasing of frequency band in term of power percentage is evaluated for desynchronization/synchronization of the ERD/ERS brain signals. As prior to movement commencement, mu-ERD can be notable over sensory-motor cortex. Meanwhile, the beta-ERD as the beta band contralateral to the movement and beta-ERS ipsilateral [18]. The gammafrequency band gave an information of maximum oscillations shortly before beginning of movement and while movement execution. It is shown as in Fig. 2.

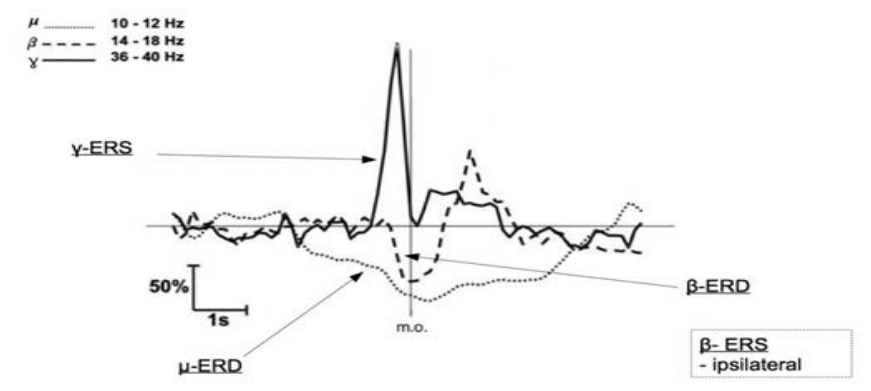

Fig. 2. The Classical Evaluation of ERD/ERS have Categorized the Brain Signal Into three Frequency Bands which are Mu-Band (10-12 Hz), BetaBand $(14-30 \mathrm{~Hz})$ and Gamma-Band $(30-40 \mathrm{~Hz})[22]$.

\section{EXPERIMENTAL METHODOLOGY}

\section{A. Neurosky's Mindwave Mobile Set-up}

The acquisition of EEG data is being done by Neurosky's headset. The headset is connected firstly to Open VIBE acquisition server so the BCI system designed by Open VIBE can be deployed. The "COM Port" need to be set correctly. The "Play" button is clicked and if the connection is successful, the acquisition signal will be displayed as in Fig. 3. As Neurosky's implemented dry electrode, it does not need to be moisturized.

\section{B. Design of the Motor Imagery BCI System}

Graz Motor Imagery is a standard of the mental state discrimination paradigm based on event related potentials. This system is designed by depending on the visually presented cue stimulus. It also based on discrete feedback which involving a symbol of cross ("+") that will be presented at the center of the monitor screen. Depending on the cue stimulus, the subject's task is to focus on their mental states for Motor Imagery to be classified into four control inputs to be visualized in OpenViBE (Right, Left, Up and Down) as in Fig. 4.

The user was asked to concentrate and to fixate to the computer monitor where the fixation cross will starting to be presented at the center of the monitor screen including a short 'beep' cue tone. An arrow will overlaid the fixated cross at the center of the monitor, pointing either between 4 directions. Subject or users will be given an instruction so that the subject is able to have four different movement imaginations. The direction of the arrow at the monitor interface will depends on it.

To train the classifier that will discriminate Right, Left, Up and Down direction by ERD/ERS brain signals, the EEG data is needed to be acquired An online acquisition scenario is developed to detect both Motor Imagery stimulus and record regular EEG brain signal data. It will acquire the brain signal and all the data needed to train classifier and spatial filter to detect ERD/ERS signal for Motor Imagery system. The data collected from this online acquisition scenario can be used to other developed scenarios. A Common Spatial Pattern (CSP) trainer scenario is developed to compute spatial filter coefficients according to the Common Spatial Pattern algorithm. A third offline developed scenario is used to train the classifier to differentiate for ERD/ERS signal classification. The trained classifier finally being applied to the online Motor Imagery scenario created.

There are 3 Classifiers that can be employed in the scenario which are Linear Discriminant Analysis (LDA), Support Vector Machine (SVM) and Multilayer Perceptron. However, in this study, SVM classifier is implemented. SVM classifier used to differentiate between Motor Imagery signal classes during the scenario online. A probability value provided by SVM classifier will show where the signal belongs in classes [23]. Prior to the classification, before proceeding to the "classifier trainer" box in Classifier Trainer Scenario, preprocessed of the data is done by filtering the data to frequency between 8-30 Hz. Then, the data is decimated and segmented into epoch. Data features extraction is done by the epoch of EEG data acquire being used for trained the classifier. Prior to classifier trainer scenario, same process being through for 
trained Common Spatial Pattern (CSP) spatial filter. Before training for the CSP spatial filter, it should first filter the data with respect to the desired band which is $8-30 \mathrm{~Hz}$. As stated from Open VIBE, CSP spatial filter is only implemented for two classes [16]. Hence, the implementation that had been done is to have two CSP spatial filter trainer boxes to train four epochs classes. Then the Stimulation multiplexer box algorithm merges several stimulation streams into one stimulation stream. The CSP algorithm work by minimized one condition of signal variance and increasing the other condition.

As for the Online Motor Imagery BCI system, the trained CSP spatial filter and classifier is employed in real time for it to be used online. The "Feature aggregator" box allows these features to be aggregated into a feature vector. Then, the classification step is done by using the "Classifier" box which is implemented with the trained classifier from the previous scenario. The processed data is sent to "Matrix Transpose" box and "Graz Visualization" box. Finally, the "Matrix Transpose" output is sent to the "LSL export (Gipsa)" box that interact by establishing communication with Python after the processed data being translated into a command for controlling the robots. The instruction for the subject to start Motor Imagery is generated by the "Graz Motor Imagery BCI Simulator" box at the early stage in this real-time online scenario.

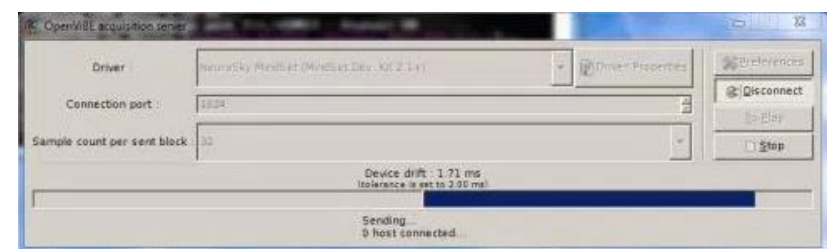

Fig. 3. OpenVibe acquisition Server Connected with Neurosky's Mindwave Mobile.

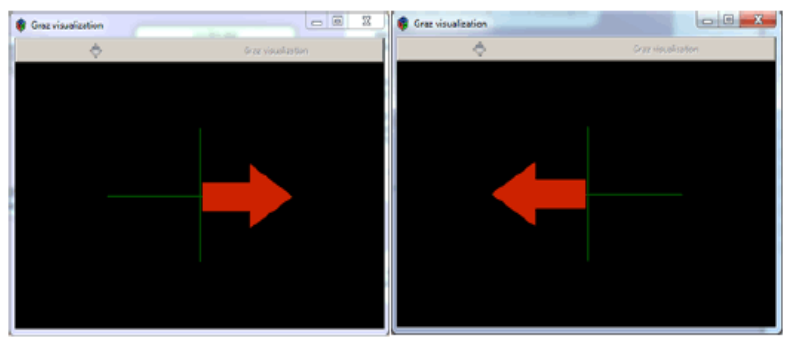

Fig. 4. Graz Motor Imagery Stimulation.

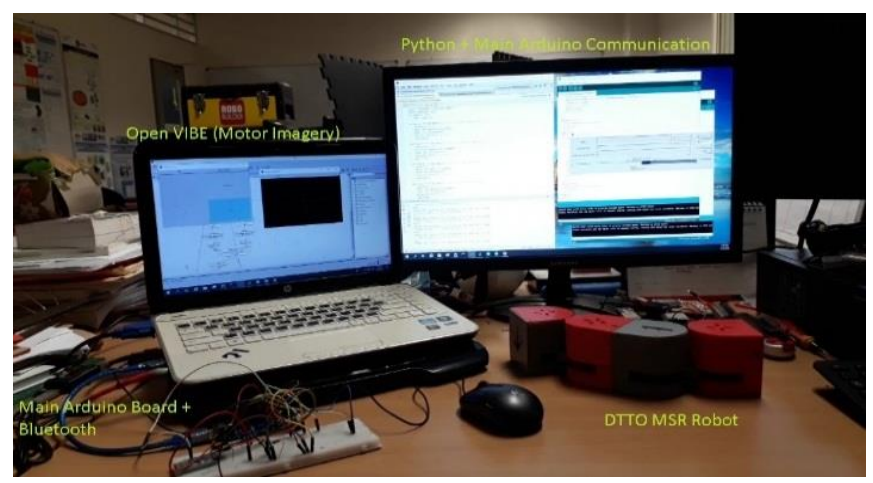

Fig. 5. Experimentation Setup (Neurosky's Headset had been Wear).
The Dtto MSR robot is supposed to have 4 configurations, thus, 4 control inputs are needed for MI BCI system to determine the configurations for the Dtto MSR robot to change. The main idea is to associate the classified control output to the configuration number for the Dtto MSR robot.

As mentioned, the output data from OpenViBE being sent to the "LSL export (Gipsa)" box that interact by establishing communication with Python. Python programming language is needed in this implementation as there is no other way for direct communication between OpenViBE to Arduino unless there is intermediary programming language. A while-loop concept of Python coding is designed so the communication between Python and V-REP is maintained. The multiple robot configurations are able to change from time to time based on the input for the predetermined modular robot configuration. Those configurations were established by maintaining Python coding loop without changing the coding every time the configuration is changing. Then, the data from Python will be sent to main Arduino board. Bluetooth is being established so the main Arduino will transmit the data wirelessly to Dtto MSR robot and propagate multiple configurations. Fig. 5 shows the experimentation setup for controlling MSR robot by EEG brain signals.

\section{PERformance's Result AND Discussion}

The data collected is based on 2 subjects that control the robot which are before training to control the robot (Untrained) and after training to control the robot (Trained). Hence, there are 4 performance results. Each data is based on 30 trials with predetermined configuration for the user to control the robot. The gender of the user is same as well as the age. It is because, to ensure this study is not influenced by gender or age factor. Based on Graz Motor Imagery generated based on EEG, the Left arrow represent Left hand movement for 1st configuration. The Right arrow represents Right hand movement for 2 nd configuration. The Up arrow represents Left foot movement for 3rd configuration and the Down arrow represent Right foot movement for 4th configuration. This study is focusing on the establishment of EEG control for the Dtto robot to propagate multiple configurations. The modular robot control attempt results are summarized in Fig. 6.

\section{Control Attempt Results}

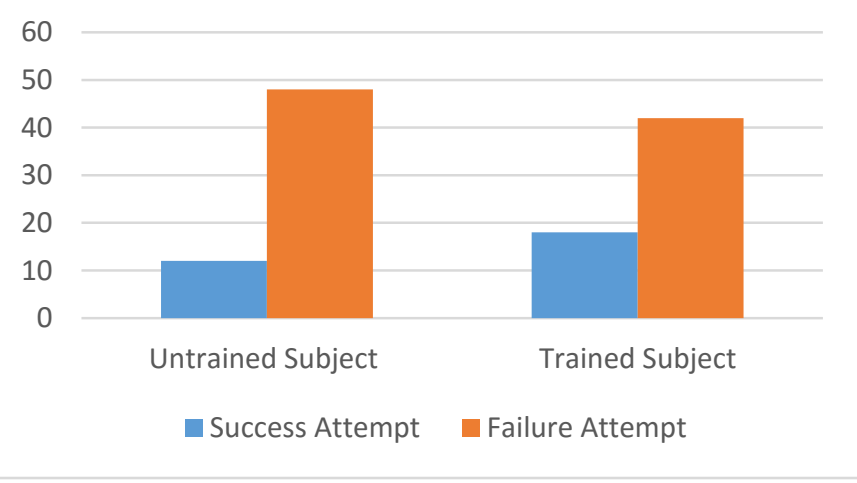

Fig. 6. Results for Dtto Robot Controlled by Brain. 
Two 19 years old subject is participate in this study as EEG user where both of them are untrained subject initially. After the first experiment, both subject being trained for Motor Imagery. In this Motor Imagery study, the Motor Imagery system used is Cue-based BCI manner. This is mainly due to the asynchronous type is not depending to the cue-based manner of BCI system, so it might produce the output control signal that results based on the users unintended control as undefined mental task will be classified as control signals. They were asked for 30 control attempts of the robot where the configuration of the robot is based on the configuration instructed. As conclusion, the results obtained as in Fig. 6 (for both subjects) shows that the success rate for untrained subjects is $20 \%$ and the success rate for trained subject is $30 \%$. In term of both subjects, the results in Fig. 6 can be detailed for both subject (Subject A and Subject B). The result is shown in Fig. 7.

Regarding the results of Fig. 6 and Fig. 7, the failures can be mainly resulted from the signal quality. It is because of the EEG device equipment where the signal acquisition is done by Neurosky's Mindwave Mobile device. As the BCI system developed is Motor Imagery system where ERD/ERS brain signal is exploited, the used of Neuroky's EEG where it only have one electrode may not be really suitable for Motor Imagery system. Unless for a system that only exploited the signal for concentration and meditation, it is necessary to use an EEG acquisition device where there are several electrodes, place on the head scalp based on 10-20 electrode placement. Besides that, the results could also be affected by how good the subject is trained. We can see a little improvement in the result of trained subject compared to untrained subject. Compared to P300 and SSVEP brain signals, Motor Imagery require an extensive training. Basically, trained subject give a better results as they are becoming better in concentration. Even though the subject is being trained, the results might be improved better if other type EEG acquisition device is being used.

Basically, the overall system is based around data transmission from OpenViBE to Dtto robot (master and slave). These data are transferred then trigger each command. If the right signal trigger is achieved, then if the failure still happened, it might be due to Noise error or Robot error. For better understanding, the results of modular robot configuration control can be seen in Fig. 8.

Fig. 8 shows the percentage of successful attempt with the indication of error probability. The $3 \%$ of Noise error happened as it involves the communication among the robot. As Dtto robot use NRF24L01 for communication with each other, noise error might happen during communication and the robot unable to propagate intended configuration. For $8 \%$ robot error, it happened as the robot unable to move at all. It might due to servo or low power supply for the robot that need to be further investigate.

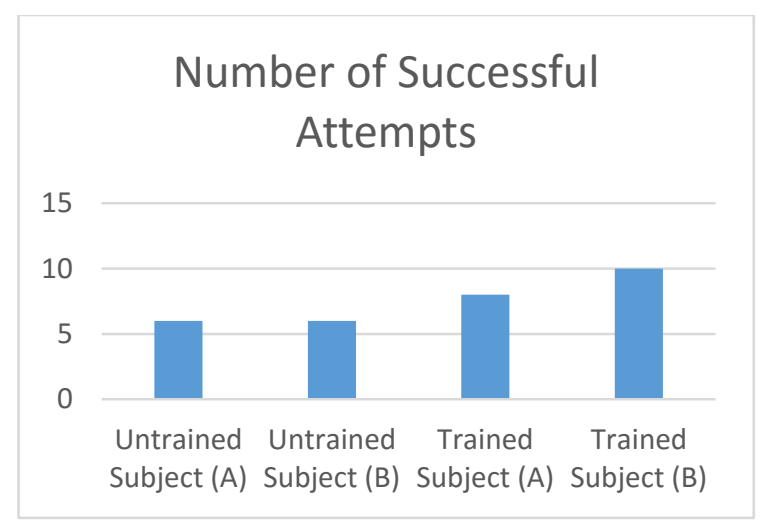

Fig. 7. Results for Trained and untrained Subjects.

\section{Results of Brain-controlled DTTO robot}

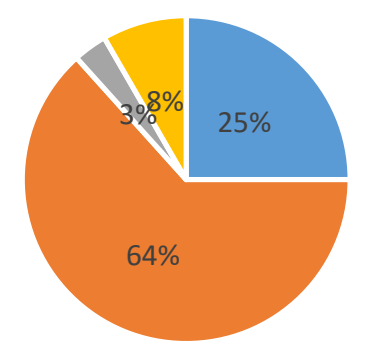

$$
\begin{aligned}
& \text { - Successful Attempt } \quad \text { Failed Motor Imagery } \\
& \square \text { Noise Error } \quad \text { Robot Error }
\end{aligned}
$$

Fig. 8. Results for Modular Robot Configuration Control.

Besides that, for the Dtto robot ability of change the configuration based on EEG brain signal, the subject instructed the robot to change the robot configuration based on the Motor Imagery. All the robot configurations desired is shown as in Fig. 9, Fig. 10, Fig. 11 and Fig. 12 as follows.

The results of this experiment (out of number of successful attempt) in term of robot multiple configurations can be seen in Table I.

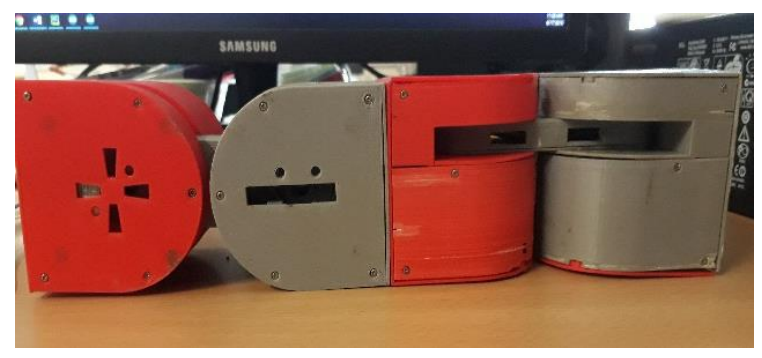

Fig. 9. Desired $1^{\text {st }}$ Configuration (Straight Line-up). 


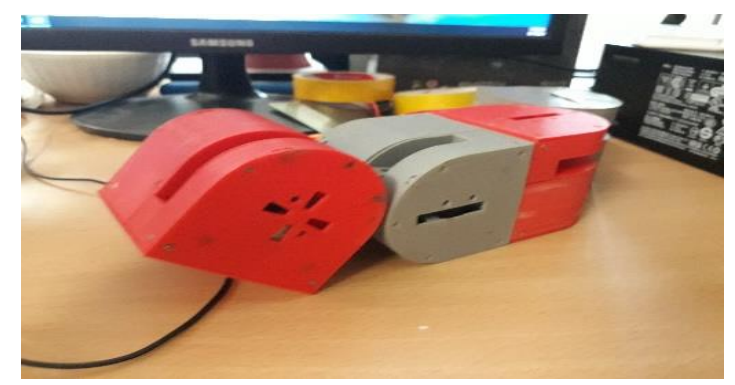

Fig. 10. Desired $2^{\text {nd }}$ Configuration (Snake-like Propagation).

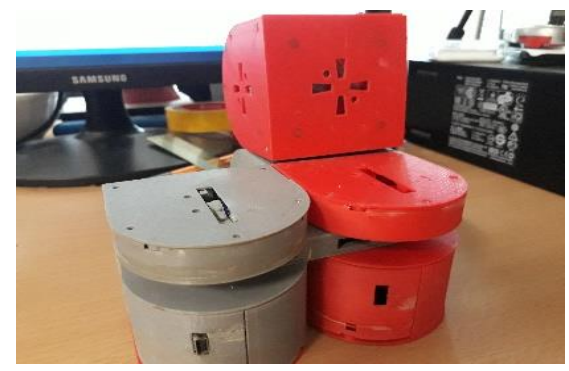

Fig. 11. Desired $3^{\text {rd }}$ Configuration (Compact-shaped).

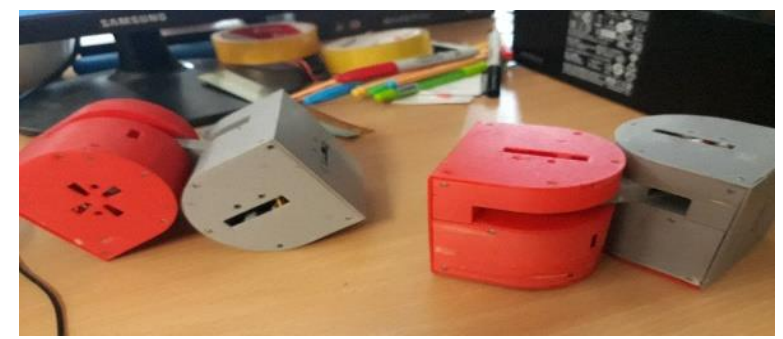

Fig. 12. Desired 4th Configuration (Separataion of Modular Robot).

TABLE I. SUCCESSFUl ATtEMPTS IN TERM OF ROBOT RECONFIGURATION

\begin{tabular}{|c|c|c|c|c|}
\hline \multirow{2}{*}{ Subjects } & \multicolumn{4}{|c|}{$\begin{array}{l}\text { Issued Commands (based on Successful Attempt within } \\
\text { the Subjects) }\end{array}$} \\
\hline & $\begin{array}{l}\text { Configuratio } \\
n A(\%)\end{array}$ & $\begin{array}{l}\text { Configurati } \\
\text { on } B(\%)\end{array}$ & $\begin{array}{l}\text { Configurati } \\
\text { on } C(\%)\end{array}$ & $\begin{array}{l}\text { Configurati } \\
\text { on } D(\%)\end{array}$ \\
\hline $\begin{array}{l}\text { Untrained } \\
\text { Subject A }\end{array}$ & 33.33 & 0 & 33.33 & 33.33 \\
\hline $\begin{array}{l}\text { Untrained } \\
\text { Subject B }\end{array}$ & 16.67 & 33.33 & 50.00 & 0 \\
\hline $\begin{array}{l}\text { Trained } \\
\text { Subject A }\end{array}$ & 37.50 & 25.00 & 25.00 & 12.50 \\
\hline $\begin{array}{l}\text { Trained } \\
\text { Subject B }\end{array}$ & 30.00 & 30.00 & 20.00 & 20.00 \\
\hline
\end{tabular}

This data shows that even though the efficiency for Neurosky's EEG device to control multiple Dtto MSR robot is low, it is possible to control MSR robot with brain as shown by the result in Table I. Most of the configurations are able to be achieved by the MSR robot when instructed by the subjects through the developed BCI system. It also able to change multiple type of configurations as intended by user through brain control.

The result obtained in this study is as expected and it might be due to a limitation. The available EEG device during this study is the Neurosky's Mindwave headset. Even though it is possible to obtain a better EEG device, we believe that as a preliminary study on the Brain-controlled MSR robot subject, the result will help us to obtain the necessary information and it will be used for future research work. Besides that, the other limitation is the number of MSR Dtto robot used in this study. In this study, only 2 modules of Dtto robot are available to be used in our experiment. Each configuration can be in more complex shape if more robot modules are being used.

Overall, the communication system establishment from OpenViBE to Dtto robot can be said as successful with low error probability in term of Noise error and Robot error. The high probability of error in term of Motor Imagery signal is based on the ERD/ERS brain signal acquirement. However, the performance obtained for Motor Imagery BCI system may be improved by using OpenViBE-compatible EEG acquisition device and with the subject that undergo intensive Motor Imagery training. Hence, it is possible for MSR robot to be controlled by brain and perform multiple configurations.

\section{CONCLUSION}

Most BCI system implemented to robot is for the robot control, which the command according to Motor Imagery. Results produced in this study provide an insight of implementing BCI system to multiple MSR robots for changing multiple configurations. It can be concluded that the potential for implementation of BCI and MSR robot can be achieved. Even though there are still many technical problems with the robot and the existing BCI system, it still has the potential to be successful in future, and the research and development for both of it are still ongoing for further improvement. Although the Motor Imagery BCI system may executes more than two commands, as MSR robot, more command to be executed should be a lot more advantages for MSR robot to have more configurations to be propagated. P300 or SSVEP might make it possible as they may offer more degree of freedom.

Besides that, the system designed is employed by a lowcost EEG headset device which still proves that the communication from OpenViBE to Dtto robot can be established. The data recorded from EEG device is obtained in real time. Experiments with subjects shows the framework introduced can achieve a better result in multiple MSR robot control in real time.

Overall, the objective of this paper to show that multiple MSR robots can be controlled by brain is achieved even though the results of the experiments are not really good as expected. However, the results show that the establishment of control communication between OpenVibe to Python language for robot control is achievable.

\section{FUTURE WORK}

There are some improvements that are possible to do for future work. Motor Imagery scenario could be improved to obtained better result. Besides that, there could be a more efficient way possible to establish communication from OpenViBE to Dtto MSR robot. Besides that, the number of robot module will be added in future research work so that it can be prove that the system is capable to control a lot more robot module to perform more complex configurations. 


\section{REFERENCES}

[1] J. J. Vidal, "Toward Direct Brain-Computer Communication," Annual Review on Biophysics and Bioengineering, vol. 2, pp. 57-80, 1973.

[2] X. Hou, Y. Liu, O. Sourina, Y. R. E. Tan, L. Wang and W. MuellerWittig, "EEG based Stress Monitoring," IEEE International Conference on Systems, Man, and Cybernetics, pp. 3110-3115, 2015.

[3] M. X. Cohen, "Where Does EEG Come From and What Does It Mean?," Trends Neuroscience, vol. 40, no. 4, pp. 208-218, 2017.

[4] Z. Tang, S. Sun, S. Zhang, Y. Chen, C. Li and S. Chen, "A BrainMachine Interface Based on ERD/ERS for an Upper-Limb Exoskeleton Control," Sensors, vol. 16, no. 12, pp. 1-14, 2016.

[5] H. Al-Negheimish, L. Al-Andas, L. Al-Mofeez, A. Al-Abdullatif, N. AlKhalifa and A. Al-Wabil, "Brainwave typing: Comparative study of P300 and motor imagery for typing using dry-electrode EEG devices, International Conference on Human-Computer Interaction, pp. 569-573, 2013.

[6] A. Malki, C. Yamg, N. Wang, and Z. Li, "Mind Guided Motion Control of Robot Manipulator Using EEG Signals," $20155^{\text {th }}$ International Conference on Information Science and Technology (ICIST), pp. 6, 2015.

[7] Q. Bin Zhao, L. Q. Zhang, and A. Cichoki, "EEG-based Asynchronous BCI control of a car in 3D Virtual Reality Environments," Chinese Science Bulletin, vol. 54, no. 1, pp. 78-87, 2009.

[8] F. Lotte, H. Mouchere, and A. Lécuyer, "attern rejection strategies for the design of self-paced eeg-based brain-computer interfaces," International Conference on Pattern Recognition, pp. 1-5, 2008.

[9] G. Pfurtscheller, T. Solis-Escalante, R. Ortner, P. Linortner, and G. R. Muller-Putz, "Self-paced operation of an SSVEP-based orthosis with and without an imagery-based 'brain switch': A feasibility study towards a hybrid BCI," IEEE Transactions on Neural Systems and Rehabilitation Engineering, vol. 18, no. 4, pp. 409-414, 2010.

[10] A. Satti, D. Coyle, and G. Prasad, "Continuous EEG classification for a self-paced BCI," 2009 4th International IEEE/EMBS Conference on Neural Engineering, NER '09, pp. 315-318, 2009.

[11] G. Ala, G. Fasshauer, E. Francomano, S. Ganci and M. McCourt, "The Method of Fundamental Solutions in Solving Coupled Boundary Value Problems for M/EEG," SIAM Journal on Scientific Computing, vol. 37, no. 4, pp. 570-590, 2015.
[12] D. Cohen, and E. Halgren, "Magnetoencephalography," Encyclopedia of Neuroscience. Oxford: Academic Press, pp. 615-622, 2009.

[13] J. Ward, “The Student's Guide to Cognitive Neuroscience," Psychology, Press Release, University of Rochester, 2000.

[14] P. D. Girase, and M. P. Deshmukh, "Mindwave Device Wheelchair Control," International Journal of Science and Research (IJSR), vol. 5, no. 6, pp. 2172-2176, 2016.

[15] K. V. Singala and K. R. Trivedi, "Connection Setup of Openvibe Tool with EEG Headset, Parsing and Processing of EEG signals," International Conference on Communication and Signal Processing (ICCSP), pp. 902-906, 2016.

[16] Y. Renard, F. Lotte, G. Gibert, M. Congedo, E. Maby, V. Delannoy, O. Bertrand, and A. Lecuyer, "OpenViBE: An Open-Source Software Platform to Design, Test, and Use Brain-Computer Interfaces in Real and Virtual Environments," Presence Teleoperators Virtual Environment, vol. 9, no. 1, pp. 35-53, 2010.

[17] Alberto, "Dtto - Explorer Modular Robot," Hackaday, 2016. [Online]. Available: https://hackaday.io/project/9976-dtto-explorer-modularrobot. [Accessed: 04-Jul-2017].

[18] G. Pfurtscheller, and F. H. Lopes, "Event-related EEG / MEG synchronization and desynchronization: basic principles," Clinical Neurophysiology, vol. 110, no. 11, pp. 1842-1857, 1999.

[19] D. R. Freitas, A. V. Inocêncio, L. T. Lins, E. A. Santos and M. A. Benedetti, "A Real-Time Embedded System Design for ERD/ERS Measurement on EEG-Based Brain-Computer Interfaces," XXVI Brazilian Congress on Biomedical Engineering, pp.25-33, 2019.

[20] L. Manca, "Master Thesis Proposal: Comparison between ERD / ERS and MRCP based movement prediction on EEG-data," 2013.

[21] C. Neuper, and G. Pfurtscheller, "Evidence for distinct beta resonance frequencies in human EEG related to specific sensorimotor cortical areas," Clinical Neurophysiology, vol. 112, no. 11, pp. 2084-2097, 2001.

[22] P. J. Durka, "From wavelets to adaptive approximations: time-frequency parametrization of EEG. Biomedical Engineering Online," vol. 2, no. 1, pp. 1, 2003.

[23] K. R. Muller, M. Tangermann, G. Dornhege, M. Krauledat, G. Curio, and B. Blankertz, "Machine learning for real-time single-trial EEGanalysis: From brain - computer interfacing to mental state monitoring," Journal Neuroscience Methods, vol. 167, no. 1, pp. 82-90, 2008. 